\title{
Case for diagnosis: A particular form of a common disease
}

\author{
Sara Elloudi, Mariame Meziane, Salim Gallouj, Fatima Zahra Mernissi
}

Department of Dermatology and Venerology. University Hospital Hassan II, FEZ, Morocco

Corresponding author: Dr. Sara Elloudi, E-mail: saraelloudi@gmail.com

Sir,

A 28-years- old man, with history of psoriasis vulgaris and psoriatic arthritis, consulted our department for skin lesions having a particular morphology like showed in picture. Several diagnoses were mentioned. Skin examination, showed erythematous plaques well limited, to regular contours, rounded, with varying sizes and colors on the face, trunk and limbs. These plaques covered with thick, circular, concentric and conical scales (Fig. 1). Psoriasis was suspected and confirmed by skin biopsy (Fig. 2). Considering this conical form of lesions typically resembling shells limpet, we have confirmed a rare form of psoriasis: Psoriasis rupioid. The patient responded well to topical treatment, the methotrexate been indicated for psoriasis arthritis.

Psoriasis may present intensely hyperkeratotic lesions, thus being classified as rupioid, ostraceous and elephantine psoriasis. These varieties are extremely rare, with few reports in the literature.

The first, more precise descriptions of the rupioide and ostreacea variants of psoriasis were made by polish dermatologist Marian Grzybowski in 1948. The rupioid forme is characterized by hyperkeratotic, circular concentric layers of scale that create a cone (rupia). Lesions with firmly adhered thick scales, varying color and a very characteristic concave surface resembling an oyster shell are typical features of ostraceous psoriasis.

Another infrequent hyperkeratotic form of psoriasis is elephantine psoriasis, which is characterized by the presence of thick and flat, long-lasting, extensive plaques. Lesions typically are found on the back, upper limbs, and buttocks, and often are extensive. Patients with thick plaque psoriasis are more likely to be male

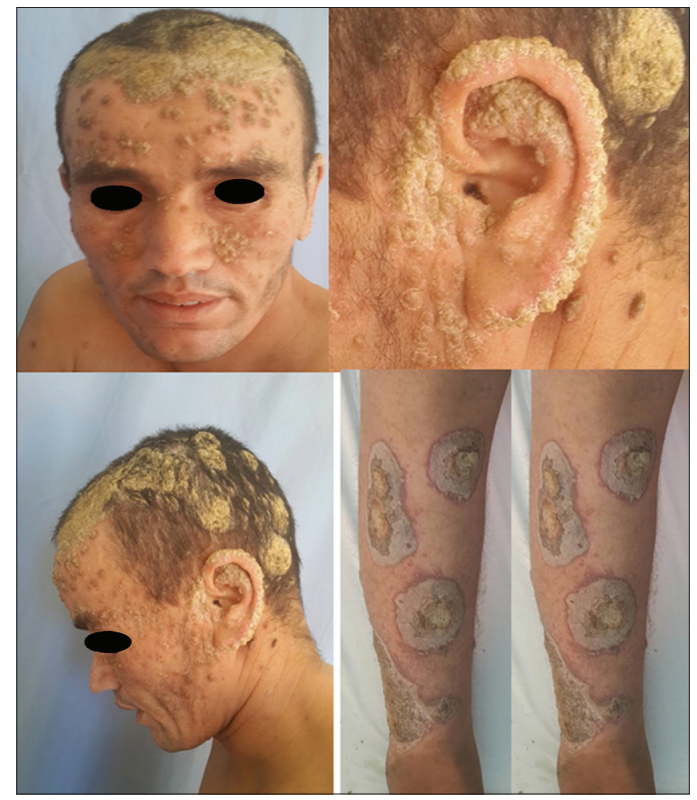

Figure 1: Plaques covered with thick, circular, concentric and conical scales on the scalp, face, ears and leg like shells limpet.

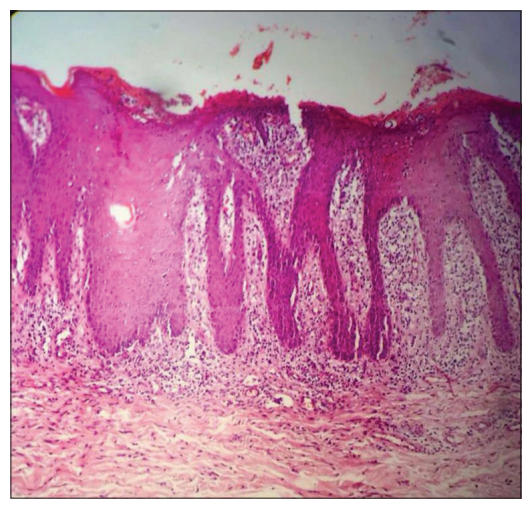

Figure 2: Microscopic examination of a hematoxylin and eosin-stained specimen revealed epidermal thickening with wide rete ridges as well as elongated papillae of the dermis with elongated and enlarged vascular plexuses. Parakeratosis of the horny layer with a Munro microabscess, regular, atrophy of the granular layer, thickening of the spinous layer. (H\&Ex100).

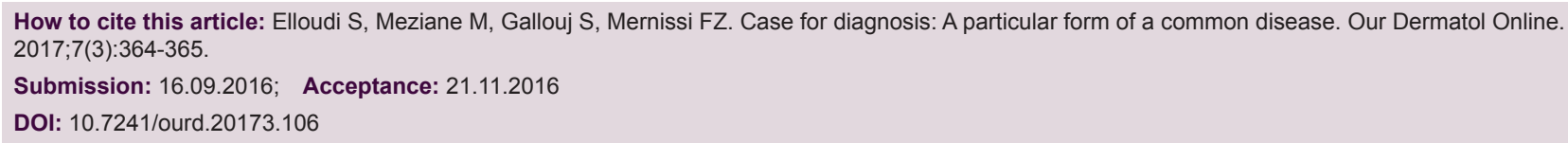


with a higher incidence of nail disease and psoriatic arthritis as well as a greater body surface area affected than patients with thin plaque psoriatic. Although most cases of rupioid psoriasis were associated with psoriatic arthritis, our patient had psoriasis arthritic without nail changes $[1,2]$.

Most of these forms are resistant to topical treatment, possibly because of the hyperkeratosis present in the lesions. Our patient responded well to topical treatment even before the introduction of methotrexate for his psoriatic arthritis.

Our case represents a rare underappreciated form of psoriasis that may mimic other skin conditions. A skin biopsy is useful in helping the clinician make the correct diagnosis.

\section{REFERENCES}

1. Chung HG, Marley-Kemp D, Keller M. Rupioid Psoriasis and Other Skin Diseases With Rupioid Manifestations. Cutis. 2014;94:11921.

2. Murakami T, Ohtsuki M, Nakagawa H. Rupioid psoriasis with arthropathy. Clin Exp Dermatol. 2000;25:409-12.

Copyright by Sara Elloudi, et al. This is an open-access article distributed under the terms of the Creative Commons Attribution License, which permits unrestricted use, distribution, and reproduction in any medium, provided the original author and source are credited.

Source of Support: Nil, Conflict of Interest: None declared. 